\title{
Superioniclike Diffusion in an Elemental Crystal: bcc Titanium
}

\author{
D. G. Sangiovanni, ${ }^{1,2, *}$ J. Klarbring, ${ }^{1}$ D. Smirnova,${ }^{2,3}$ N. V. Skripnyak, ${ }^{1}$ D. Gambino, ${ }^{1}$ \\ M. Mrovec, ${ }^{2}$ S. I. Simak, ${ }^{1}$ and I. A. Abrikosov ${ }^{1}$ \\ ${ }^{1}$ Department of Physics, Chemistry and Biology (IFM) Linköping University, SE-581 83 Linköping, Sweden \\ ${ }^{2}$ ICAMS, Ruhr-Universität Bochum, D-44780 Bochum, Germany \\ ${ }^{3}$ Joint Institute for High Temperatures, Russian Academy of Sciences, 125412 Moscow, Russia
}

(Received 15 March 2019; revised manuscript received 20 June 2019; published 3 September 2019)

\begin{abstract}
Recent theoretical investigations [A. B. Belonoshko et al. Nat. Geosci. 10, 312 (2017)] revealed the occurrence of the concerted migration of several atoms in bcc Fe at inner-core temperatures and pressures. Here, we combine first-principles and semiempirical atomistic simulations to show that a diffusion mechanism analogous to the one predicted for bcc iron at extreme conditions is also operative and of relevance for the high-temperature bcc phase of pure $\mathrm{Ti}$ at ambient pressure. The mechanism entails a rapid collective movement of numerous (from two to dozens) neighbors along tangled closed-loop paths in defect-free crystal regions. We argue that this phenomenon closely resembles the diffusion behavior of superionics and liquid metals. Furthermore, we suggest that concerted migration is the atomistic manifestation of vanishingly small $\omega$-mode phonon frequencies previously detected via neutron scattering and the mechanism underlying anomalously large and markedly non-Arrhenius self-diffusivities characteristic of bec Ti.
\end{abstract}

DOI: 10.1103/PhysRevLett.123.105501

Mass transport in elemental crystals is primarily regulated by migration of intrinsic point defects such as vacancies and self-interstitials. Vacancies and selfinterstitials, present in dilute concentrations at equilibrium, are generated or annihilated at, e.g., surfaces and grain boundaries, or via Frenkel-pair formation and recombination in defect-free lattice regions. The diffusion of individual point defects is often well described by uncorrelated hops among vicinal stable positions, which allows evaluation of diffusivities via simple stochastic-walk models. However, the occurrence of more complex migration mechanisms in elemental crystals has also been proposed. For example, experimental and theoretical studies indicated that self-interstitials propagate extremely rapidly in bcc metals $[1,2]$ due to the long-range compressive strain that they produce in $\langle 111\rangle$ lattice rows (defects known as crowdions [3-5]). Other diffusion mechanisms proposed for pure metals include the spontaneous concerted exchange of two, or more, atoms in a defect-free lattice. In particular, in 1950, theoretical results by Zener indicated that the synchronized cyclic motion of four atoms in fcc copper (four-atom ring mechanism) is energetically more favored than the direct exchange of two neighbors [6].

Among elemental crystals, group-IVB bcc transition metals have attracted significant interest due to large atomic self-diffusivities $D$ and sharply non-Arrhenius trends in $D$ vs inverse temperature [7]. The mechanism(s) that control diffusion in group-IVB bcc metals have long been under debate [8]. Hypotheses made to explain the anomalous temperature dependence of self-diffusion coefficients include the mixture of diffusion mechanisms and temperature-induced modifications of the effective activation energy for a single migration pathway [9]. In this regard, tracer-diffusivity experiments [10] excluded the possibility of four-atom ring diffusion (proposed in Ref. [6]) controlling mass transport in bcc Ti and Ti-Nb alloys. Nonetheless, Gibbs et al. [10] asserted that the occurrence of diffusion reactions involving the concerted motion of $\geq 8$ Ti neighbors would be consistent with their measurements.

Complex diffusion mechanisms are known for exotic types of materials such as superionic conductors [11-14], superheated crystals [15], and supercooled liquids $[14,16,17]$. While specific details of the mechanism in these cases differ, they all entail the distinctively concerted migration of several atoms, often referred to as stringlike or liquidlike diffusion. Recent theoretical investigations by Belonoshko et al. [18] identified concerted migration in bcc Fe subject to core temperatures and pressures. The results of Ref. [18] are intriguing, as they suggest that rapid concerted diffusion may be a phenomenon of relevance even in elemental crystals. Nevertheless, the experimental observation of liquidlike atomic migration in bcc Fe is practically unfeasible due to the extreme conditions necessary to dynamically stabilize this metallic phase [18]. Here, we combine classical and ab initio molecular dynamics (CMD and AIMD) to demonstrate that highly collective diffusion processes are active at temperatures safely below melting in the bcc phase of pure Ti at ambient pressure. We show that the occurrence of the concerted migration, i.e., simultaneous diffusion of $\geq 2$ atoms in defect-free lattice 
regions, may resolve long-standing questions concerning the mass transport and phonon properties of group-IVB bcc metals.

AIMD [19] simulations are carried out using VASP [20] implemented with the projector augmented-wave method [21], employing the Perdew-Burke-Ernzerhof [22] approximation to the electronic exchange or correlation energies. The ionic positions are updated every femtosecond, using a convergence criterion of $10^{-5} \mathrm{eV} / \mathrm{supercell}$ for the selfconsistent total energy, $2 \times 2 \times 2 k$-point grids and plane wave cutoff energies of $300 \mathrm{eV}$. AIMD simulations of defect-free bcc Ti (250 atoms) sample the canonical $N V T$ configuration space at $1800 \mathrm{~K}$, which is well above the range of bcc $\rightarrow$ hcp and bcc $\rightarrow \omega$ transition temperatures $(\approx 800-1100 \mathrm{~K})$ [23], while below the Ti melting point $T_{m} \approx 1940 \mathrm{~K}$. The temperature is controlled via the NoséHoover thermostat, using a Nosé mass corresponding to oscillations with period of 148 fs. Supercell equilibrium volumes are taken from experiments [24].

CMD simulations are performed using LAMMPS [25], describing the Ti-Ti interactions via the second-nearestneighbor modified embedded-atom method (MEAM) [26], as parametrized in Ref. [27], and integrating the equations of motion at 1-fs timesteps. The equilibrium volumes of supercells formed of $14 \times 14 \times 14$ conventional bcc cells (5488 $\mathrm{Ti}$ atoms) are determined as a function of temperature via $N P T$ sampling based on the Parrinello-Rahman barostat [28] and the Langevin thermostat. The concerted migration rates $\Gamma_{C}$, diffusivities $D_{C}$ in a defect-free lattice, and vacancy diffusivities $D_{V}$ are separately determined by combining the results obtained for Ti supercells composed of 5488 and 432 bcc sites, respectively, during $N V T$ canonical sampling at temperatures within the range 0.73-0.97T $T_{m}$ [29]. Details on the evaluation of $D_{V}, \Gamma_{C}$, and $D_{C}$ with corresponding activation energies $E_{a}$, attempt frequencies $\nu$, and prefactors $D_{0}$ are given in Ref. [30]. The MEAM potential, carefully parametrized via matching the model forces with AIMD forces [27], correctly reproduces the mechanical instability of bcc Ti at low temperatures, as well as hcp $\rightarrow$ bcc transformations, but is unable to describe the phonon instability which drives spontaneous bcc $\rightarrow \omega$ transitions at $0 \mathrm{~K}$. This, however, is not expected to qualitatively affect the conclusions of this work, which are based on results obtained at temperatures much higher than bcc $\rightarrow \omega$ transition temperatures.

To estimate equilibrium vacancy and self-interstitial concentrations $\left(c_{V}, c_{\mathrm{SI}}\right)$ and total self-diffusion coefficients $D_{\text {tot }}$ [33] as a function of temperature $T$, we employ the approach proposed in Ref. [34] for a (initially defect-free) bcc Ti(001) surface slab (165600-atom supercell). CMD simulations show that point defects in the slab interior are generated by thermal fluctuations (Frenkel pairs) or had originally formed at the surface. The open surfaces allow maintaining the equilibrium balance between different defects [30].
In addition to the validation procedure performed in Ref. [27], the reliability of MEAM for the description of bcc $\mathrm{Ti}$ properties is established by comparing CMD vs experimental phonon dispersion curves $[35,36]$ and total diffusion coefficients $D_{\text {tot }}$ [9,37-39]; see Ref. [30]. The temperature-dependent effective potential (TDEP) method [40-42] is used to calculate the phonon spectra of bcc Ti (5488-atom supercell) by fitting second order force constants to displacement and force datasets extracted from CMD simulations. Videos [30] and snapshots are produced using visual molecular dynamics [43].

Figure 1 illustrates concerted migration events in defectfree bcc Ti recorded during AIMD at $1800 \mathrm{~K}$. The figure is a superposition of snapshots in which atom color variations are indicative of time progression during $9 \mathrm{ps}$. Two separate processes are identified: (i) the direct exchange of two neighbors, which lasts for $<1 \mathrm{ps,}$, and (ii) the concerted migration of 22 neighbors along a tangled closed-loop trajectory, with duration of $\approx 9$ ps. The path intersects supercell boundaries and follows both $\langle 111\rangle$ (nearestneighbor) and $\langle 100\rangle$ (next-nearest-neighbor) directions.

CMD simulations carried out for 5488-atom defect-free bcc Ti supercells at temperatures between 0.73 and $0.97 T_{m}$

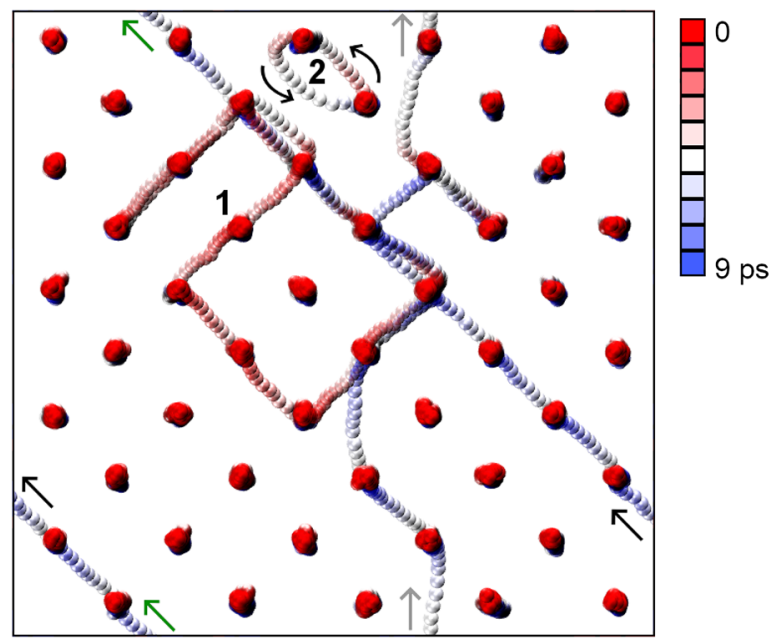

FIG. 1. Orthographic (001)-plane view of overlapped AIMD snapshots taken during the concerted migration in defect-free bcc Ti at $1800 \mathrm{~K}$. In each snapshot, atoms are colored according to time progression during $\approx 9 \mathrm{ps}$ (see legend). Identified events are labeled as "1" (concerted migration of 22 atoms) and " 2 " (Ti-pair exchange). The complex mechanism 1 entails that a tangled closed-loop atomic chain-with $\langle 111\rangle$ and $\langle 001\rangle$ chain segments linking nearest-neighbors and next-nearestneighbors-glides in a cyclic fashion within the solid host crystal. During the reaction, each chain atom replaces the next on the chain sequence. Straight colored arrows facilitate following atomic displacements across supercell boundaries. For clarity, trajectory smoothing over 1000 steps is used to reduce the vibrational noise. Simulation videos and additional description of concerted diffusion mechanisms (Sec. S5) are provided in Supplemental Material [30]. 
complement the information provided by AIMD simulations at $1800 \mathrm{~K}\left(0.93 T_{m}\right)$. In agreement with AIMD observations, CMD runs show that the reaction pathways for the concerted Ti migration include Ti-pair exchange, four-atom ring rotation, or synchronized migration of dozens of neighbors (see Sec. S5 of Supplemental Material for additional illustration [30]). The extended concerted diffusion events are initiated with a $\mathrm{Ti}$ atom that, by pushing a neighbor, triggers a chain of migration reactions. The reaction duration scales with the number of atoms involved; the ideal lattice is broken and reformed within $\sim 10^{-13}-10^{-12} \mathrm{~s}$ (Ti-pair exchange) up to $\approx 1 \mathrm{~ns}$ (collective motion of dozens of atoms). Although the concerted migration phenomena as, e.g., the cyclic motion of few atoms, are believed to control diffusion in liquid and superheated metals $[15,44]$, this type of mass transport process has not been previously reported-except for bcc Fe at inner-core conditions [18]—in elemental solid crystals below the melting point.

The activation energy and attempt frequency of the concerted migration evaluated via Arrhenius linear regression of CMD rates in defect-free cells [30] are $E_{a C}=$ $3.1 \pm 0.2 \mathrm{eV}$ and $\nu_{C}=1.0\left(\times 5.2^{ \pm 1}\right) \times 10^{15} \mathrm{~s}^{-1}$ atom $^{-1}$ [see inset of Fig. 2(a)]. The probability for one lattice atom to trigger collective motion at $1800 \mathrm{~K}$ $\left(2.1 \times 10^{6 \pm 0.3} \mathrm{~s}^{-1}\right.$ atom $\left.^{-1}\right)$ is reasonably consistent with the estimate $\left(\approx 10^{8 \pm 1.5} \mathrm{~s}^{-1}\right.$ atom $\left.{ }^{-1}[45]\right)$ of a single AIMD run $(250$ atoms $)$ at the same temperature. Although the concerted migration rates exhibit Arrhenius-like behavior, the slope of the corresponding self-diffusion coefficients $D_{C}(T)$ significantly varies with the inverse temperature [orange squares in Fig. 2(a)]. For $T$ increasing from $\approx 0.73$ to $0.97 T_{m}$, the $\log \left[D_{C}(T)\right]$ vs $T^{-1}$ curve changes concavity (upward $\rightarrow$ downward) at $\approx 0.85 T_{m}$. Simulations performed for supercells that contain one vacancy [green circles in Fig. (2a)] show that the concerted diffusion also enhances vacancy transport; the collective motion of a $\langle 111\rangle$ atomic chain formed of $n$ nearest neighbors produces a net vacancy translation by $n$ lattice positions. For migration of individual vacancies, we obtain activation energies $E_{a V}=0.19 \pm 0.02 \mathrm{eV}$ and prefactors $D_{0 V} c_{V}^{-1}=$ $6.2\left(\times 1.2^{ \pm 1}\right) \times 10^{-8} \mathrm{~m}^{2} \mathrm{~s}^{-1}$ [red hexagons in Fig. 2(b)], where $c_{V} \approx 0.2 \%$ is kept constant with $T$. The contribution of vacancy migration to the total diffusivity becomes progressively less important as $T$ increases. This is qualitatively demonstrated in Fig. 2(a), which shows that the difference between self-diffusion coefficients in 5488-site supercells with (green circles) and without (orange squares) defects becomes vanishingly small near $T_{m}$.

The highly correlated motion of several $\mathrm{Ti}$ neighbors over a relatively flat energy landscape $(\approx 0.2 \mathrm{eV}$ barrier for vacancy migration) resembles the liquidlike diffusion properties characteristic of superionics (migration energies $\approx 0.2-0.3 \mathrm{eV}$ ) [46]. Also, the trend in $\log \left[D_{C}(T)\right]$ vs $T^{-1}$ determined for defect-free bcc $\mathrm{Ti}$ [orange squares in
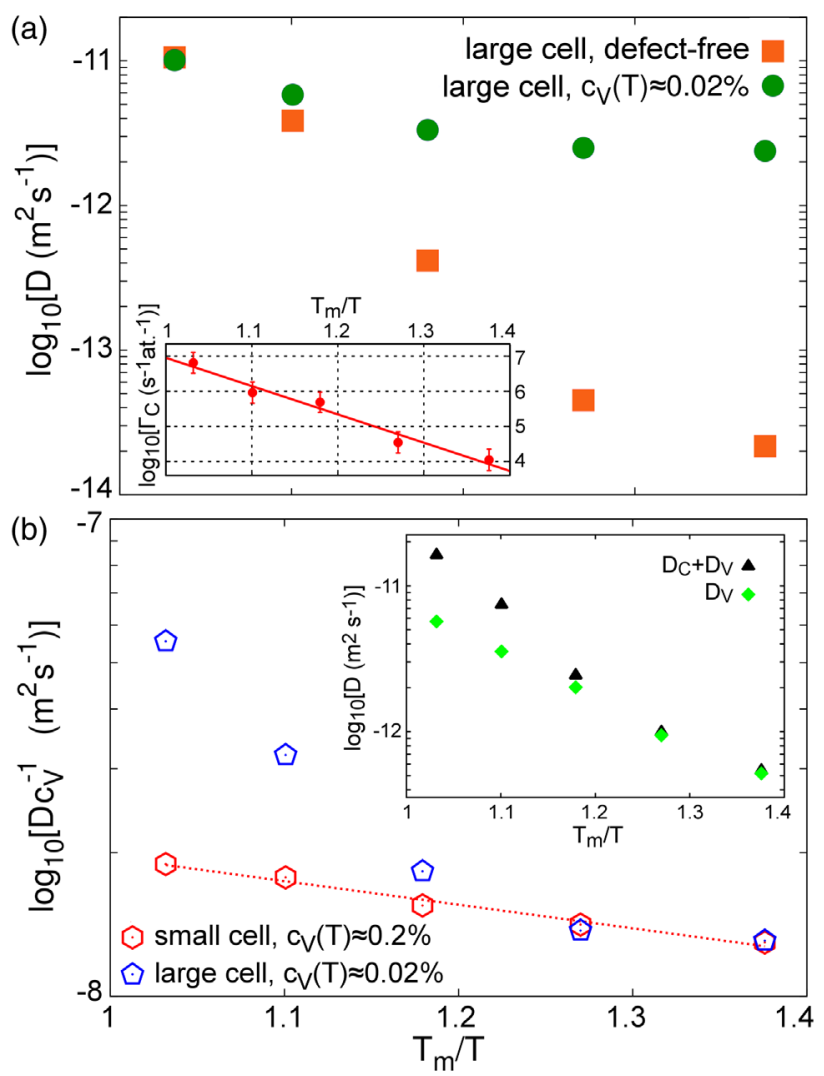

FIG. 2. Temperature dependence of Ti self-diffusion due to vacancy and/or collective atomic motion determined via CMD. The labels large (small) indicate results of supercells containing 5488 (432) bcc lattice sites. (a) The supercells contain one vacancy (green circles) or no defects (orange squares, $D_{C}$ ). The inset illustrates the temperature dependence of the concerted migration rates $\Gamma_{C}$. (b) Vacancy-concentration-normalized diffusion coefficients as a function of supercell size; both large and small supercells contain one vacancy. In smaller cells (red hexagons), diffusion is exclusively due to the vacancy migration (no diffusion in defect-free regions). The concerted migration in defect-free regions of larger cells (blue pentagons) yields significant diffusivities for $T_{m} / T<1.2$. The inset shows results for $D_{V}$ [diffusivity of an individual vacancy multiplied by $\left.c_{V}(T)\right]$ vs total self-diffusivities (approximated as $D_{C}+D_{V}$ ) [30].

Fig. 2(a)] is qualitatively similar to that reported for bulk anion diffusivities in the type-II superionics $\mathrm{CaF}_{2}$ and $\mathrm{UO}_{2}$ (note the change in the curvature for temperatures approaching superionic transitions in Fig. 7 of Ref. [12]).

Superionic conductors are classified as type I or type II, respectively, depending on whether or not the transition to the disordered superionic state is accompanied by a structural transformation [47]. The diffusion behavior in bcc $\mathrm{Ti}$ is reminiscent of the one observed in type-II superionics in that the concerted migration becomes dominant at $T>0.8 T_{m}$ [11] and no structural transition is associated with the onset of high diffusivity. At variance with superionics, where liquidlike diffusion is present in one sublattice while the remaining system stays ordered, 
bcc $\mathrm{Ti}$ shows localized liquidlike diffusion while also acting as a solid host crystal.

The spontaneous formation of Frenkel defects (vacancy and self-interstitial pairs) is considered of central importance for promoting liquidlike diffusion in type-II superionics [47] and superheated crystals [15]. In particular, Zhang et al. [15] noted that interstitial defects serve as initiators of stringlike diffusion in superheated $\mathrm{Ni}$ and that the concerted process itself can be viewed as the propagation of interstitials along the string. It may be possible to make a similar interpretation of the concerted migration in bec $\mathrm{Ti}$, although analyzing this in terms of energyquenched CMD snapshots, as in Ref. [15], is not possible here due to the dynamical instability of the bcc structure at $0 \mathrm{~K}$ [27]. We can, however, make some observations regarding the energetics of defect formation. Calculated vacancy equilibrium concentrations follow an Arrhenius trend within the investigated temperature range [see Fig. $2 \mathrm{~S}$ of Ref. [30] ]. Linear regression of $\log \left[c_{V}(T)\right]$ and $\log \left[c_{\mathrm{SI}}(T)\right]$ vs $T^{-1}$ (Table I) yields free energies of formation $G_{V}^{f}=1.00 \pm 0.05 \mathrm{eV}$ and $G_{\mathrm{SI}}^{f} \approx 3.5 \mathrm{eV}$. The fact that $G_{\mathrm{SI}}^{f}+G_{V}^{f} \approx 4.5 \mathrm{eV}$ is much greater than the activation energy $E_{a C}(=3.1 \pm 0.2 \mathrm{eV})$ indicates that the concerted diffusion in bcc $\mathrm{Ti}$ is a more complex process than the static formation of a Frenkel pair followed by rapid vacancy and self-interstitial migration and recombination.

Our observation of concerted migration may resolve several long-standing questions concerning mass transport and phonon properties of bcc $\mathrm{Ti}$ and, possibly, bec $\mathrm{Zr}$ and Hf. Experimental studies attributed the anomalies in mass transport properties of group-IVB transition metals to features in their vibrational spectra [7]. Special attention was dedicated to analyzing the effects of soft phonon modes. Neutron scattering measurements indicated that the primary migration mechanism in bcc Ti is a simple $\langle 111\rangle$ nearest-neighbor vacancy jump [48]. Rapid vacancy migration is enabled by soft $2 / 3\langle 111\rangle$ longitudinal modes [ $\omega$ mode, Fig. 3(a)], a characteristic of bcc-structure groupIVB metals [35,36,49,50], which assist atomic displacements along $\langle 111\rangle$ directions [36].

The $\omega$ mode brings closer pairs of adjacent (111) lattice planes while leaving unaltered positions in every third (111) atomic layer [Figs. 3(b) and 3(c)]. $\omega$ vibrations also correspond to parallel adjacent $\langle 111\rangle$ atomic rows (strings) sliding one onto another maintaining unvaried interatomic

TABLE I. Equilibrium concentration of vacancies and selfinterstitials in bcc Ti estimated via CMD.

\begin{tabular}{lcc}
\hline \hline$T_{m} / T$ & $c_{V}$ & $c_{\mathrm{SI}}$ \\
\hline 1.38 & $(4.0 \pm 0.1) \times 10^{-5}$ & $\ldots$ \\
1.27 & $(6.7 \pm 0.1) \times 10^{-5}$ & $\ldots$ \\
1.18 & $(1.3 \pm 0.1) \times 10^{-4}$ & $(1.3 \pm 0.1) \times 10^{-5}$ \\
1.10 & $(2.0 \pm 0.1) \times 10^{-4}$ & $(6.7 \pm 0.1) \times 10^{-5}$ \\
\hline \hline
\end{tabular}

spacing within each string [Figs. 3(b) and 3(c)]. The $\omega$-mode softness in group-IVB bcc metals originates from weak interstring forces (due, in turn, to $d$-electron screening) and electron accumulation along individual strings [7,51]. The observation of long $\langle 111\rangle$ atomic chains rapidly gliding against each other, Fig. 1, suggests that the concerted migration is a consequence of soft $\omega$ modes. $\omega$-like environments act as transition states for collective atomic motions characterized by continuous bec $\rightarrow \omega \rightarrow$ bec local structural changes [Fig. 3(c)].

The upward curvature in self-diffusivities $\log [D(T)]$ vs $T^{-1}$, observed upon cooling bcc Ti toward the martensitic transformation temperature, has been correlated with the progressive softening of transversal $T_{1} 1 / 2\langle 110\rangle$ phonon modes, which lowers the vacancy migration enthalpy by causing distortion of the saddle-point configuration [7]. We note, however, that the curvature in $\log \left[D_{V}(T)\right]$ vs $T^{-1}$ $\left[D_{V}(T) \propto c_{V}(T) \Gamma_{V}(T)\right.$, where $\Gamma_{V}(T)$ are vacancy jump frequencies] may also arise from non-Arrhenius trends in vacancy equilibrium concentrations $c_{V}(T)$ [52]. While experimental studies focused on understanding the breakdown of the Arrhenius law for $T$ approaching martensitic transitions, our results provide clarification for trends and magnitudes of $\mathrm{Ti}$ self-diffusivities at temperatures above $0.7 T_{m}$.

Present CMD results rule out both scenarios of $c_{V}(T)$ (Fig. S2 [30]) and $\Gamma_{V}(T)$ [red hexagons in Fig. 2(b)]
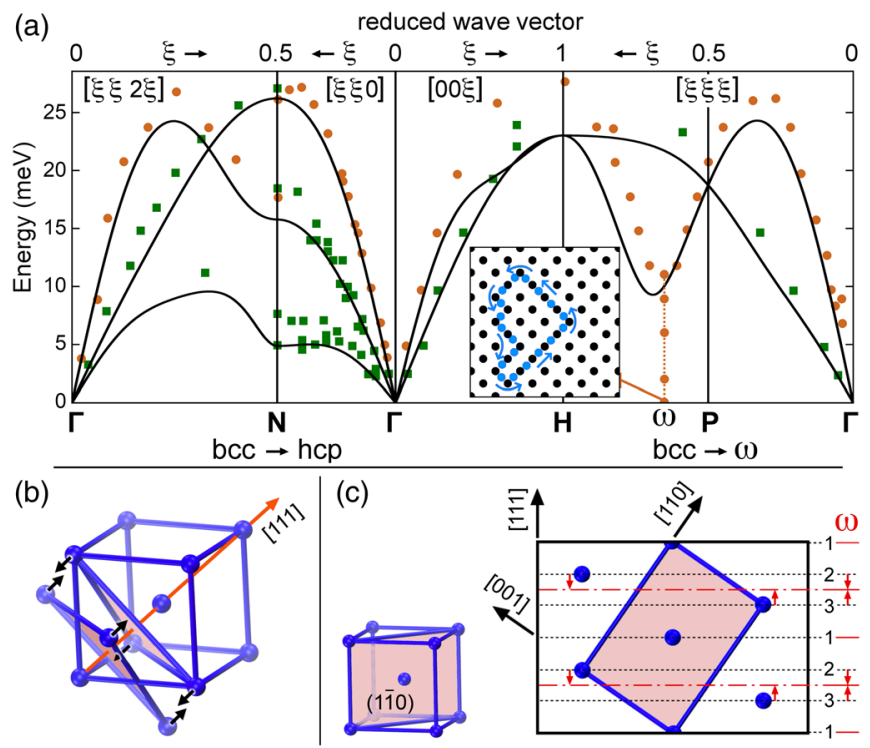

FIG. 3. (a) Titanium phonon dispersion evaluated via CMD (solid lines) vs inelastic neutron scattering data at $\approx 1300 \mathrm{~K}$. Experimental longitudinal- and transversal-mode frequencies are marked by orange circles and green squares, respectively. Note that experiments $[35,36]$ report different frequencies for $T_{1}$ modes along $\Gamma \rightarrow \mathrm{N}$ paths. The inset in (a) is a schematic illustration of liquidlike diffusion, here suggested to underlie anomalously low $\omega$ frequencies in bcc Ti. (b),(c) Schematic representation of longitudinal 2/3[111] $\omega$ phonons responsible for bcc $\rightarrow \omega$ phase transitions and the concerted migration in titanium. 
displaying non-Arrhenius behavior for $T>0.7 T_{m}$. Figure S3 of Ref. [30] demonstrates that the total diffusion coefficients $D_{\text {tot }}(T)$ evaluated via simulations of $\operatorname{Ti}(001)$ slabs are in agreement with experimental findings [9, 37-39]. A comparison of $D_{\text {tot }}(T)$ values with CMD simulation results obtained for smaller simulation boxes (details given above) reveals that the total Ti self-diffusivity is essentially due to vacancy migration for $T<0.8 T_{m}$ and concerted migration (irrespective of defect concentrations) for $T>0.8 T_{m}$. This is summarized as $D_{\text {tot }}(T) \approx D_{V}(T)+$ $\left[1-c_{V}(T)\right] D_{C}(T)$ [53], where $D_{V}(T)=c_{V}(T) D_{V}^{C_{V} \text {-indep }}(T)$ and $D_{V}^{C_{V} \text {-indep }}(T)$ is the diffusivity of an individual vacancy [30]. The concerted migration provides important contributions to mass transport at elevated $T$, which explains strong upward bending of $\log \left[D_{\text {tot }}\right]$ vs $T^{-1}$ experimentally observed for temperatures approaching $T_{m}$ [inset in Fig. 2(b) and see also Fig. S3 in Ref. [30]]. This is demonstrated, Fig. 2(b), by comparing the diffusion coefficients $D(T) c_{V}^{-1}$ for large vs small supercells (both contain one vacancy, i.e., $c_{V}(T)$ is constant). In smaller supercells, only vacancy migration contributes to $D(T)$ at all temperatures. Vacancy migration is the dominant transport mechanism for large cells at relatively low temperatures [note hexagons and pentagons overlap for $T_{m} / T>1.2$, see Fig. 2(b)], while the frequent occurrence of the concerted migration in defect-free lattice regions for $T_{m} / T<1.2$ produces an upward curvature in $\log \left[D(T) c_{V}^{-1}\right]$ vs $T_{m} / T$.

In addition to providing fundamental understanding for Ti mass transport properties, the occurrence of liquidlike diffusion clarifies puzzling features observed in bcc $\mathrm{Ti}$ phonon spectra $[35,36]$. CMD simulations well reproduce the phonon frequencies determined via neutron scattering measurements on bcc Ti, Fig. 3(a). In agreement with experiments, CMD phonon dispersions exhibit no appreciable variation in $\omega$-mode frequencies and reproduce the softening of $T_{1} 1 / 2\langle 110\rangle$ modes upon lowering $T$ toward martensitic transition temperatures (Fig. S4 [30]). In Fig. 3(a), one can note that the experiments detect a wide range of phonon energies (these reach vanishingly small values) in correspondence to the $\omega$ mode [54]. We argue below that such a peculiarity is due to the occurrence of the concerted migration.

The experimental study in Ref. [48] discussed possible origins for zero-frequency $\omega$ modes detected by neutron scattering in bcc Ti. Petry et al. [48] excluded the presence of static $\omega$-structure embryos and noted, instead, that (i) $\omega$ phonons have lifetimes of $\sim 10^{-13} \mathrm{~s}$ and (ii) no coherent elastic intensity is detected for $\hbar \omega=0$ at $\mathbf{q}=2 / 3\langle 111\rangle$. In this regard, Petry et al. [35] stated that a loss in real-space correlation combined with short phonon lifetimes is indicative of a liquidlike behavior in a localized region (near the $\omega$ point) of reciprocal space. Since atomic diffusion corresponds to zero-frequency vibrational modes of the system [55] and stringlike concerted migration closely relates with atomic displacements produced by $\omega$ phonons [Fig. 3(b)], we suggest that the concerted diffusion is the atomistic manifestation of the peculiar feature observed in bcc $\mathrm{Ti}$ phonon spectra [inset in Fig. 3(a)].

We can make yet another analogy between the behavior of bcc $\mathrm{Ti}$ and type-II superionics. A particular phonon mode of $B_{1 u}$ symmetry appears to be connected to the superionic behavior in type-II superionic transitions [56-60]. The atomic displacements in this mode are closely connected to diffusion processes in fluorite crystal structures, analogous to the case of $\omega$-mode displacements or the concerted migration in bcc Ti. Similar to the Ti $\omega$ mode, the $\mathrm{B}_{1 u}$ mode is not critically soft in TDEP descriptions [60], but is so in the quasiharmonic case.

To summarize, atomistic simulations show that highly concerted stringlike diffusion mechanisms are active in bcc $\mathrm{Ti}$ at temperatures below melting. Such diffusion processes have been previously observed for exotic materials, like superionic conductors and superheated crystals, or for iron under extreme temperatures and pressures. We propose that this diffusion mechanism may address fundamental questions concerning anomalies in mass transport and phonon properties of bcc Ti. Given the similarities in the properties of group-IVB metals, the concerted migration is expected to be operative in bcc $\mathrm{Zr}$ [49] and $\mathrm{Hf}$ [50].

All simulations were carried out using the resources provided by the Swedish National Infrastructure for Computing (SNIC), on the clusters located at the National Supercomputer Centre (NSC) in Linköping, on the Beskow cluster located at the Center for High Performance Computing (PDC) in Stockholm, and on the Kebnekaise cluster located at the High Performance Computing Center North (HPC2N) in Umeå, Sweden. D. G. S. gratefully acknowledges financial support from the Olle Engkvist Foundation. Financial support from the Swedish Research Council (VR) through Grants No. 2015-04391 and No. 2014-4750, the Swedish Government Strategic Research Area in Materials Science on Functional Materials at Linköping University (Faculty Grant SFOMat-LiU No. 2009-00971), and the VINN Excellence Center Functional Nanoscale Materials (FunMat-2) Grant No. 2016-05156 is gratefully acknowledged.

*Corresponding author. davide.sangiovanni@liu.se

[1] T. Amino, K. Arakawa, and H. Mori, Activation energy for long-range migration of self-interstitial atoms in tungsten obtained by direct measurement of radiation-induced pointdefect clusters, Philos. Mag. Lett. 91, 86 (2011).

[2] K. Urban and A. Seeger, Radiation-induced diffusion of point-defects during low-temperature electron irradiation, Philos. Mag. 30, 1395 (1974).

[3] H. R. Paneth, The mechanism of self-diffusion in alkali metals, Phys. Rev. 80, 708 (1950). 
[4] P. M. Derlet, D. Nguyen-Manh, and S. L. Dudarev, Multiscale modeling of crowdion and vacancy defects in body-centered-cubic transition metals, Phys. Rev. B 76, 054107 (2007).

[5] P.-W. Ma and S. L. Dudarev, Universality of point defect structure in body-centered cubic metals, Phys. Rev. Mater. 3, 013605 (2019).

[6] C. Zener, Ring diffusion in metals, Acta Crystallogr. 3, 346 (1950).

[7] C. Herzig, The correlation between diffusion behavior and phonon softening in bcc metals, Ber. Bunsen-Ges. Phys. Chem. 93, 1247 (1989).

[8] C. Herzig, U. Kohler, and S. V. Divinski, Tracer diffusion and mechanism of non-Arrhenius diffusion behavior of $\mathrm{Zr}$ and $\mathrm{Nb}$ in body-centered cubic $\mathrm{Zr}-\mathrm{Nb}$ alloys, J. Appl. Phys. 85, 8119 (1999).

[9] U. Kohler and C. Herzig, On the anomalous self-diffusion in bcc titanium, Phys. Status Solidi B 144, 243 (1987).

[10] G. B. Gibbs, D. Graham, and D. H. Tomlin, Diffusion in titanium and titanium-niobium alloys, Philos. Mag. 8, 1269 (1963).

[11] S. Hull, Superionics: Crystal structures and conduction processes, Rep. Prog. Phys. 67, 1233 (2004).

[12] A. Annamareddy and J. Eapen, Low dimensional string-like relaxation underpins superionic conduction in fluorites and related structures, Sci. Rep. 7, 44149 (2017).

[13] V. A. Annamareddy, P. K. Nandi, X. Mei, and J. Eapen, Waxing and waning of dynamical heterogeneity in the superionic state, Phys. Rev. E 89, 010301(R) (2014).

[14] A. Gray-Weale and P. A. Madden, Dynamical arrest in superionic crystals and supercooled liquids, J. Phys. Chem. B 108, 6624 (2004).

[15] H. Zhang, M. Khalkhali, Q. Liu, and J. F. Douglas, Stringlike cooperative motion in homogeneous melting, J. Chem. Phys. 138, 12A538 (2013).

[16] C. Donati, S. C. Glotzer, P. H. Poole, W. Kob, and S. J. Plimpton, Spatial correlations of mobility and immobility in a glass-forming Lennard-Jones liquid, Phys. Rev. E 60, 3107 (1999).

[17] X. P. Tang, U. Geyer, R. Busch, W. L. Johnson, and Y. Wu, Diffusion mechanisms in metallic supercooled liquids and glasses, Nature (London) 402, 160 (1999).

[18] A. B. Belonoshko, T. Lukinov, J. Fu, J. Zhao, S. Davis, and S. I. Simak, Stabilization of body-centered cubic iron under inner-core conditions, Nat. Geosci. 10, 312 (2017).

[19] R. Car and M. Parrinello, Unified Approach for Molecular Dynamics and Density-Functional Theory, Phys. Rev. Lett. 55, 2471 (1985).

[20] G. Kresse and J. Furthmuller, Efficient iterative schemes for ab initio total-energy calculations using a plane-wave basis set, Phys. Rev. B 54, 11169 (1996).

[21] P. E. Blöchl, Projector augmented-wave method, Phys. Rev. B 50, 17953 (1994).

[22] J. P. Perdew, K. Burke, and M. Ernzerhof, Generalized Gradient Approximation Made Simple, Phys. Rev. Lett. 77, 3865 (1996).

[23] Z.-G. Mei, S.-L. Shang, Y. Wang, and Z.-K. Liu, Densityfunctional study of the thermodynamic properties and the pressure-temperature phase diagram of Ti, Phys. Rev. B 80, 104116 (2009).
[24] X. G. Lu, M. Selleby, and B. Sundman, Assessments of molar volume and thermal expansion for selected bcc, fcc and hcp metallic elements, CALPHAD: Comput. Coupling Phase Diagrams Thermochem. 29, 68 (2005), and references therein.

[25] S. Plimpton, Fast parallel algorithms for short-range molecular dynamics, J. Comput. Phys. 117, 1 (1995).

[26] B. J. Lee and M. I. Baskes, Second nearest-neighbor modified embedded-atom-method potential, Phys. Rev. B 62 , 8564 (2000).

[27] W.-S. Ko, B. Grabowski, and J. Neugebauer, Development and application of a Ni-Ti interatomic potential with high predictive accuracy of the martensitic phase transition, Phys. Rev. B 92, 134107 (2015).

[28] M. Parrinello and A. Rahman, Polymorphic transitions in single crystals: A new molecular dynamics method, J. Appl. Phys. 52, 7182 (1981).

[29] Experimental and CMD results are compared as a function of normalized temperatures $T_{m} / T$ : The Ti melting point is $\approx 1940 \mathrm{~K}$; MEAM estimates $T_{m}=1651 \mathrm{~K}$ [27].

[30] See Supplemental Material, which includes Refs. [31,32], at http://link.aps.org/supplemental/10.1103/PhysRevLett.123 .105501 for (i) Results of lattice thermal expansion and phonon spectra as a function of $T$. (ii) Methods used for evaluation of vacancy, concerted migration, and total $\mathrm{Ti}$ self-diffusion coefficients, vacancy and self-interstitial equilibrium concentrations. (iii) Detailed description of the concerted migration processes. (iv) Videos of CMD and AIMD simulations.

[31] D. Gambino, D. G. Sangiovanni, B. Alling, and I. A. Abrikosov, Nonequilibrium $a b$ initio molecular dynamics determination of Ti monovacancy migration rates in $B 1 \mathrm{TiN}$, Phys. Rev. B 96, 104306 (2017).

[32] D. E. Smirnova, S. V. Starikov, and I. S. Gordeev, Evaluation of the structure and properties for the high-temperature phase of zirconium from the atomistic simulations, Comput. Mater. Sci. 152, 51 (2018).

[33] We show below that $D_{\text {tot }}$ is well approximated as the sum of vacancy $D_{V}(T)$ and concerted migration $D_{C}(T)$ diffusion coefficients which are separately determined via CMD simulations of 432- and 5488-bcc-site supercells. The actual total diffusivities are accurately determined via $\mathrm{Ti}(001)$ slab simulations [30].

[34] M. I. Mendelev and Y. Mishin, Molecular dynamics study of self-diffusion in bcc Fe, Phys. Rev. B 80, 144111 (2009).

[35] W. Petry, A. Heiming, J. Trampenau, M. Alba, C. Herzig, H. R. Schober, and G. Vogl, Phonon dispersion of the bcc phase of group-IV metals. 1. bcc titanium, Phys. Rev. B 43, 10933 (1991).

[36] W. Petry, A. Heiming, J. Trampenau, M. Alba, and G. Vogl, Strong phonon softening in the bcc phase of titanium, Physica (Amsterdam) 156B-157B, 56 (1989).

[37] J. F. Murdock, T. S. Lundy, and E. E. Stansbury, Diffusion of $\mathrm{Ti}^{44}$ and $\mathrm{V}^{48}$ in titanium, Acta Metall. 12, 1033 (1964).

[38] N. E. Walsöe De Reca and C. M. Libanati, Self diffusion in beta titanium and beta hafnium, Acta Metall. 16, 1297 (1968).

[39] A. E. Pontau and D. Lazarus, Diffusion of titanium and niobium in bcc Ti-Nb alloys, Phys. Rev. B 19, 4027 (1979). 
[40] O. Hellman, I. A. Abrikosov, and S. I. Simak, Lattice dynamics of anharmonic solids from first principles, Phys. Rev. B 84, 180301(R) (2011).

[41] O. Hellman, P. Steneteg, I. A. Abrikosov, and S. I. Simak, Temperature dependent effective potential method for accurate free energy calculations of solids, Phys. Rev. B 87, 104111 (2013).

[42] O. Hellman and I. A. Abrikosov, Temperature-dependent effective third-order interatomic force constants from first principles, Phys. Rev. B 88, 144301 (2013).

[43] W. Humphrey, A. Dalke, and K. Schulten, VMD: Visual molecular dynamics, J. Mol. Graphics Modell. 14, 33 (1996).

[44] I. V. Belova, T. Ahmed, U. Sarder, A. V. Evteev, E. V. Levchenko, and G. E. Murch, The Manning factor for direct exchange and ring diffusion mechanisms, Philos. Mag. 97, 230 (2017).

[45] The uncertainty on the AIMD rate is assessed from the scatter in $\Gamma_{C}(T)$ recorded from several CMD simulations at a given $T$.

[46] X. He, Y. Zhu, and Y. Mo, Origin of fast ion diffusion in super-ionic conductors, Nat. Commun. 8, 15893 (2017).

[47] A. K. Sagotra, D. Errandonea, and C. Cazorla, Mechanocaloric effects in superionic thin films from atomistic simulations, Nat. Commun. 8, 963 (2017).

[48] W. Petry, T. Flottmann, A. Heiming, J. Trampenau, M. Alba, and G. Vogl, Atomistic Study of Anomalous Self-Diffusion in bcc $\beta$-Titanium, Phys. Rev. Lett. 61, 722 (1988).

[49] A. Heiming, W. Petry, J. Trampenau, M. Alba, C. Herzig, H. R. Schober, and G. Vogl, Phonon dispersion of the bcc phase of group-IV metals. 2. bcc zirconium, Phys. Rev. B 43, 10948 (1991).

[50] J. Trampenau, A. Heiming, W. Petry, M. Alba, C. Herzig, W. Miekeley, and H. R. Schober, Phonon dispersion of the bcc phase of group-IV metals. 3. bcc hafnium, Phys. Rev. B 43, 10963 (1991).

[51] K. M. Ho, C. L. Fu, and B. N. Harmon, Microscopic analysis of interatomic forces in transition metals with lattice distortions, Phys. Rev. B 28, 6687 (1983).

[52] A. Glensk, B. Grabowski, T. Hickel, and J. Neugebauer, Breakdown of the Arrhenius Law in Describing Vacancy Formation Energies: The Importance of Local Anharmonicity Revealed by $A b$ Initio Thermodynamics, Phys. Rev. X 4, 011018 (2014).

[53] $D_{\text {tot }}(T)$ is slightly larger than $D_{V}(T)+\left[1-c_{V}(T)\right] D_{C}(T)$ due to defect-defect interactions and $\mathrm{Ti}_{\mathrm{SI}}$ migration in slab simulations [30].

[54] TDEP is unsuited to reproduce that feature: It requires atomic trajectories to oscillate near fixed lattice positions.

[55] M. P. Desjarlais, First-principles calculation of entropy for liquid metals, Phys. Rev. E 88, 062145 (2013).

[56] L. L. Boyer, Nature of Melting and Superionicity in Alkali and Alkaline-Earth Halides, Phys. Rev. Lett. 45, 1858 (1980).

[57] J. Buckeridge, D. O. Scanlon, A. Walsh, C. R. A. Catlow, and A. A. Sokol, Dynamical response and instability in ceria under lattice expansion, Phys. Rev. B 87, 214304 (2013).

[58] M. K. Gupta, P. Goel, R. Mittal, N. Choudhury, and S. L. Chaplot, Phonon instability and mechanism of superionic conduction in $\mathrm{Li}_{2} \mathrm{O}$, Phys. Rev. B 85, 184304 (2012).

[59] J. R. Nelson, R. J. Needs, and C. J. Pickard, High-pressure $\mathrm{CaF}_{2}$ revisited: A new high-temperature phase and the role of phonons in the search for superionic conductivity, Phys. Rev. B 98, 224105 (2018).

[60] J. Klarbring, N. V. Skorodumova, and S. I. Simak, Finitetemperature lattice dynamics and superionic transition in ceria from first principles, Phys. Rev. B 97, 104309 (2018). 